\title{
Impacto de la condiciones bucales sobre la calidad de vida en escolares de ámbito rural, Piura, Perú
}

Impact of oral conditions on the quality of life in rural schoolchildren, Piura, Peru

César del Castillo-López ${ }^{1, a, c, d}$, Alexis Evangelista-Alva ${ }^{1, b, c}$, Pablo Sánchez-Borjas ${ }^{2, c}$

\section{RESUMEN}

Objetivos: El objetivo del estudio fue evaluar el impacto de las condiciones bucales en la CVRSB de niños de 11-12 años de edad, de ámbito rural de Piura. Material y Métodos: Estudio transversal, en 150 escolares, usando la versión en español (Perú) del Índice Child-OIDP para determinar el impacto de 17 condiciones bucales sobre 8 desempeños diarios. Resultados: El 88,7\% de escolares informaron uno o más de un desempeño impactado (Child-OIDP > 0). El promedio de condiciones bucales auto-percibidas como problema fue de 1,14 $\pm 0,66$ condiciones. La más frecuente fue: dolor de muela (58,0\% de escolares). Los desempeños tuvieron un promedio de severidad de impacto de $0,54 \pm 0,49$. El desempeño con mayor severidad de impacto fue comer $(1,07 \pm 0,96)$. Para el 89,5\% de escolares los impactos fueron de "poca" o "muy poca intensidad". El promedio de desempeños impactados fue 3,01 2,20. El Índice Child-OIDP promedio global fue de 7,05 7,23. Conclusión: El promedio global del Índice fue bajo en escolares rurales de Piura. No se halló diferencias significativas en los impactos según sexo o nivel educativo.

PALABRAS CLAVE: Calidad de vida, salud bucal, CVRSB, rural, Índice Child-OIDP. (DeCS, Bireme) 


\section{SUMMARY}

Objective: To evaluate the impact of oral conditions on the OHRQoL of children 11-12 years of age, of rural area of Piura. Methods: Cross sectional study of 150 school children, using a validated Spanish version (Peru) of the Child-OIDP index to determine the impact of 17 oral conditions on eight oral daily performances (Child-OIDP $>0)$. Results: The $88.7 \%$ of students reported one or more of a performance hit. The average of oral conditions self-perceived as problem was $1.14 \pm 0.66$ conditions. The most frequent was Toothache (58.0\% of students). On a scale of 0 to 3, the average Severity of impact was $0.54 \pm 0.49$. The performance with greater severity of impact was Eating (1.07 \pm 0.96). 89.5\% of the students presented Little of Very little Impact intensity. The average of impacted performances was $3.01 \pm 2.20$ performances. The Child-OIDP Index global average was 7.05 \pm 7.23 . Conclusion: Rural schoolchildren in Piura showed low level of impacts of oral conditions on OHRQoL. No significant differences were found comparing impacted performances by sex or educational level.

\section{KEYWORDS: Quality of life, oral health, OHRQoL, rural, Child-OIDP Index. (MeSH, NLM)}

\section{INTRODUCCIÓN}

Los indicadores epidemiológicos en odontología usan observaciones clínicas "objetivas" de los problemas bucales, pero no brindan información acerca del bienestar de las personas ni de la capacidad de las mismas para llevar a cabo sus roles deseados o de la satisfacción de las personas al realizar importantes actividades de la vida diaria como el masticar, hablar o dormir $(1,2)$. La Calidad de Vida Relacionada con la Salud (CVRS), o salud percibida, integra aquellos aspectos de la vida directamente relacionados con el funcionamiento físico, mental y con el estado de bienestar de las personas (3). Desde fines del siglo pasado, la medición de la CVRS se constituye en una de las mayores innovaciones en la evaluación en el campo de la salud pública (4), demostrando utilidad para decisiones desde la formulación de políticas hasta el monitoreo del tratamiento de pacientes y análisis de costo-efectividad de intervenciones en salud $(3,4)$.

En 1996, en la conferencia para evaluar las mediciones de salud bucal y calidad de vida, en Chapell Hill, se analizaron 11 indicadores para medir CVRSB $(5,6)$. En el marco conceptual, de estos indicadores se consideró la adaptación de Locker (7) de la Clasificación Internacional de Deterioros, Impedimentos y Discapacidades de la Organización Mundial de la Salud (OMS) a la odontología, estableciendo tres niveles: a) el primero, referido a la situación de salud bucal, descrito usualmente mediante indicadores clínicos; b) el segundo, referido a los impactos intermedios o primeros impactos negativos causados por las condiciones bucales (dolor, incomodidad, limitación funcional e insatisfacción con la apariencia); y, c) el tercero, referido a los impactos finales, que afectan el desarrollo de las actividades diarias de las personas, para las que se requiere adecuado rendimiento físico, psicológico y social $(5,6,7)$.

En dicha conferencia, Adulyanon y Sheiham (6) presentaron el desarrollo y la validación del Índice de Impacto Bucal en las Actividades Diarias de Adultos (Oral Impacts on Daily Performances OIDP), diseñado para medir la frecuencia y severidad de los impactos de condiciones bucales sobre ocho actividades o desempeños diarios (comer y disfrutar la comida, hablar claramente, limpieza de dientes, dormir y descansar, sonreír y reír mostrando los dientes sin vergüenza, mantener el estado emocional usual sin irritación, desempeñar el trabajo usual o rol social y contacto con la gente) correspondientes al tercer nivel del modelo de Locker $(6,7)$.

El Child-OIDP Index (Índice Child-OIDP) es la adaptación delOIDP. Fue desarrollado y validado como un cuestionario auto-administrado para relacionar la salud bucal con la calidad de vida en adolescentes, determinando impactos de 17 condiciones bucales en ocho desempeños cotidianos (impactos finales e intermedios del modelo de Locker) (8). Mide la prevalencia e intensidad de impactos y como índice expresa un valor entre 0 y 72 , indicando los valores más altos mayor alteración en la calidad de vida de los adolescentes. Ha sido traducido a varios idiomas y usado en varias poblaciones habiendo demostrado validez y buenas propiedades psicométricas (9).

El año 2007, Sánchez evaluó el impacto de las condiciones bucales sobre la calidad de vida de los 
niños de 11 y 12 años de edad en 4 instituciones educativas públicas en Zapallal, Puente Piedra y Lima; empleando la versión validada en español (Perú) del Índice Child-OIDP (10), encontrando que la prevalencia de impactos de las condiciones bucales en los desempeños diarios fue del $82,0 \%$ y el Índice alcanzó un puntaje promedio de 7,77 (11).

El objetivo del presente estudio fue determinar el impacto de las condiciones bucales sobre la CVRSB, mediante el Índice Child-OIDP, en escolares de 11 y 12 años de edad, de los distritos de Canchaque y San Miguel de El Faique de la provincia de Huancabamba, del ámbito rural de Piura, el año 2010.

\section{MATERIALES Y MÉTODOS}

El estudio fue observacional, descriptivo y transversal, en una población total de 169 escolares de 11 y 12 años de edad, matriculados en las seis instituciones educativas (I.E.) públicas escogidas por su mayor número de escolares y que a la vez eran de más fácil acceso geográfico: en el ámbito rural de Canchaque (I.E. 14477, I.E. 14478, I.E. Emilio Espinoza) y en San Miguel del Faique (I.E. 14506, I.E. 14507, I.E. S. M. Faique), del área de influencia del Centro de Salud de Canchaque y Centro de Salud San Miguel de El Faique, Huancabamba, Piura. Ambos poblados ubicados a una altura de 1,198 m.s.n.m. y 1,060 m.s.n.m., respectivamente. La población se dedica principalmente a labores del área agropecuaria doméstica.

El mínimo tamaño muestral requerido fue de 72 escolares, pero de los 169 escolares la muestra por conveniencia estuvo conformada por los 150 escolares en los que se cumplieron los criterios de inclusión (aparentemente sanos, de ambos sexos, consentimiento de los padres y asentimiento del escolar). Setentainueve fueron del nivel primaria y 71 del nivel secundaria, 61 varones y 89 mujeres. Los datos fueron recogidos, en Agosto del 2010, en dos momentos: a) primera etapa, mediante un cuestionario auto-administrado a los escolares en grupos, en aulas y horarios de cada I.E., para identificar -de una lista de 17 condiciones bucales- aquellas que constituían problema o impacto para las actividades cotidianas de cada escolar; y, b) segunda etapa, mediante entrevista estructurada individual, rostro a rostro, efectuada por el investigador (previamente capacitado por el autor del estudio en Zapallal, Puente Piedra, Lima (10), para la aplicación de la versión en español (Perú) del Índice Child-OIDP, validada previamente en Lima. La frecuencia de los impactos se obtuvo preguntándole a cada escolar, cuál (les) de las 17 condiciones bucales habían causado molestias en cada uno de los ocho desempeños habituales, y cuán a menudo en los últimos 3 meses habían sucedido. La frecuencia y la severidad fueron registradas, según el esquema usado por Gherunpong $(2,8)$ con una escala de Likert con puntajes de 1 a 3, para cada condición, según el siguiente esquema:

I) Para la frecuencia: preguntándole al escolar con qué frecuencia se presentaba la molestia, según las siguientes categorías: a) "frecuencia 1", cuando se presentaba una o dos veces al mes, b) "frecuencia 2", cuando sucedía 3 a más veces en el mes, c) "frecuencia 3", cuando sucedía 3 a más veces a la semana.

II) Para la severidad, preguntándole, cuánto la alteración bucal impactaba en el desempeño de su vida diaria, según las siguientes categorías: a) "severidad 1", cuando la molestia o impacto era poco, b) "severidad 2", cuando la molestia era moderada o regular, c) "severidad 3", cuando la molestia era considerable.

III) Para la intensidad de los impactos, se consideró al impacto con mayor grado de intensidad sobre cualquiera de los ocho desempeños en cada escolar.

Y para evaluar la intensidad en cada desempeño se consideró cinco categorías según el puntaje resultante de multiplicar severidad por frecuencia: a) "muy poca intensidad" para puntaje 1, b) "poca intensidad" para puntaje 2, c)"moderada intensidad" para puntajes de 3 a 4, d) "severa intensidad" para puntaje hasta 6, y e) "muy severa intensidad" para puntaje hasta 9 .

Los puntajes del Índice Child-OIDP, o impacto de las condiciones bucales en cada desempeño diario, se calcularon multiplicando los puntajes de frecuencia por severidad del impacto para cada desempeño habitual, en un rango de 0-9, multiplicando luego el resultado por $100 \mathrm{y}$ dividiéndolo entre 9 . El puntaje global del Î́ndice Child-OIDP por escolar se calculó sumando los puntajes delíndice en las ocho actividades, en un rango de 0-72, multiplicándolo luego por 100 y dividiéndolo entre 72. La extensión del impacto de las condiciones bucales en los desempeños se obtuvo determinando el número máximo de desempeños 
impactados por escolar, en un rango de 0-8, y para la muestra se promedió el número de desempeños impactados entre los escolares $(2,10)$.

Para el estudio se contó con la aprobación del Comité Institucional de Ética de la Universidad Peruana Cayetano Heredia, la autorización de las autoridades educativas locales, el Consentimiento Informado de los padres de familia y el Asentimiento escrito de los participantes.

\section{RESULTADOS}

En la población constituida por 169 escolares de 11 y 12 años de edad, 19 escolares no se encontraron presente en su respectiva I.E. durante los días de realización del presente estudio. Los otros 150 escolares, cumplieron los criterios de inclusión y no rechazaron su participación (ni sus padres), siendo incluidos en el estudio. Así, la muestra estuvo integrada por 150 escolares, quienes tuvieron una edad media de 11,55 \pm 0,6 años. La prevalencia de escolares con uno o más impactos de las condiciones bucales en alguno de los 8 desempeños diarios (ChildOIDP $>0$ ) fue $88,7 \%$ (133 escolares) Tabla 1. Ocho de 17 condiciones bucales fueron mencionadas como causa de impacto. Las más frecuentemente fueron: dolor de muela $(58,0 \%)$ y diente sensible $(32,7 \%)$. Las menos frecuentes fueron: fractura de diente permanente y sangrado de encías $(1,3 \%$ en cada caso), Tabla 2. Diente sensible fue significativamente más frecuente en mujeres que en varones $(p=$ 0,04). Los escolares de nivel educativo primario manifestaron con mayor frecuencia dolor de muela que los del nivel secundario $(\mathrm{p}=0,043)$; los escolares de secundaria indicaron diente sensible con mayor frecuencia que los de primaria $(\mathrm{p}=0,042)$, (Tablas 2 y 3). El promedio de condiciones bucales autopercibidas como causante de impactos fue 1,14 $\pm 0,66$ condiciones por escolar, sin diferencias significativas por sexo o por nivel educativo (Tabla 2).

En los 150 escolares la extensión de los impactos, varió de 0 a 8 desempeños con impactos. El promedio fue $3.01 \pm 2.20$ y no existió diferencias estadísticas por sexo o por nivel educativo (Tabla 3 ). Los desempeños más impactados fueron comer, limpiarse la boca $y$ emoción y los menos impactados fueron: estudiar, socializar $y$ dormir. No se encontró diferencias significativas entre sexos ni entre nivel educativo (Tabla 4).
La severidad de los impactos de las condiciones bucales sobre los desempeños diarios, tuvo un

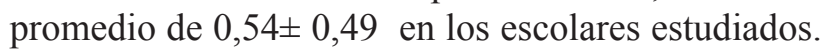
El desempeño con mayor promedio de severidad fue: comer $(1,07 \pm 0,96)$ No se halló diferencias significativas entre los promedios de severidad por desempeños ni en los promedios por sexos o por niveles educativos (Tabla 5).

Menos del uno por ciento de los escolares con impactos tuvieron intensidad de impactos severa o muy severa, y nueve de cada diez escolares con impactos tuvieron impactos con muy poca o poca intensidad, (Tabla 1). Las intensidades muy severa y severa de los impactos en cada desempeño fueron más altas en: dormir y emoción que en los otros desempeños, mientras que sonreír y estudiar fueron las más bajas. Los niveles más bajos de intensidad de impacto, muy poca y poca intensidad, fueron mayores en los desempeños: socializar y sonreir que en el resto de desempeños (Tabla 1).

El Índice Child-OIDP global, esto es el promedio de impactos bucales en los desempeños diarios, para la muestra estudiada de 150 escolares, fue 7,05 7,23. Los valores individuales en la muestra variaron en un rango de 0 a 33. Los promedios para los desempeños diarios específicos variaron entre los más altos: comer $(1,59 \pm 1,77)$ y limpiarse los dientes $(1,43 \pm$ $1,61)$, hasta los más bajos: socializar $(0,42 \pm 1,04)$ y estudiar $(0,40 \pm 0,98)$. Los valores individuales del índice variaron entre 0 a 6 en los desempeños: hablar, limpiarse los dientes, estudiar y socializar; entre 0 a 9 en los desempeños: comer, dormir, emoción y sonreír (Tabla 1).

Se determinó el índice sólo para la sub-muestra de 133 escolares con algún impacto, hallándose que el Índice Child-OIDP global fue 7,95 \pm 7,20 y los valores individuales variaron en un rango de 1 a 33 . Los promedios para los desempeños diarios específicos variaron entre los más altos: dormir $(2,64 \pm 2,00)$ y hablar $(2,52 \pm 1,50)$, hasta los más bajos: estudiar $(2,07 \pm 1,22)$ y socializar $(1,91 \pm 1,47)$. Los valores individuales del índice variaron entre 1 a 6 en los desempeños: hablar, limpiarse los dientes, estudiar y socializar, entre 1 a 9 en los desempeños: comer, dormir, emoción y sonreír (Tabla 1). 
Tabla 1. Prevalencia, intensidad y puntaje Child-OIDP de los impactos bucales en escolares de Piura.

\begin{tabular}{|c|c|c|c|c|c|c|c|c|c|}
\hline $\begin{array}{l}\text { Impactos bucales en las actividades } \\
\text { diarias }\end{array}$ & $\begin{array}{c}\text { Todas las } \\
\text { actividades } \\
\text { diarias } \\
\end{array}$ & Comer & Hablar & $\begin{array}{l}\text { Limpiarse } \\
\text { los dientes }\end{array}$ & Dormir & Emoción & Sonreir & Estudiar & Socializar \\
\hline Prevalencia (\%) & 88.7 & 62.7 & 38.7 & 60.0 & 24.7 & 40.7 & 32.7 & 20.0 & 22.0 \\
\hline $\mathrm{n}=150$ & 133 & 94 & 58 & 90 & 37 & 61 & 49 & 30 & 33 \\
\hline \multicolumn{10}{|l|}{ Puntaje de impactos $n=150$} \\
\hline Rango: mín.-máx. & $0-33$ & $0-9$ & $0-6$ & $0-6$ & $0-9$ & $0-9$ & $0-9$ & $0-6$ & $0-6$ \\
\hline Media (d.e.) & $7.05(7.23)$ & $1.59(1.77)$ & $0.91(1.51)$ & $1.43(1.61)$ & $0.63(1.49)$ & $1.01(1.67)$ & $0.65(1.33)$ & $0.40(0.98)$ & $0.42(1.04)$ \\
\hline Percentiles $(25,50,75)$ & $2,5,10$ & $0,1,2$ & $0,0,1.25$ & $0,1,2$ & $0,0,0$ & $0,0,2$ & $0,0,1$ & $0,0,0$ & $0,0,0$ \\
\hline \multicolumn{10}{|c|}{ Intensidad de impactos ( $\%$ de escolares con impactos) $n=133$} \\
\hline Muy poca & 67.7 & 33.3 & 31.5 & 30.8 & 25.0 & 34.4 & 46.9 & 37.9 & 54.5 \\
\hline Poca & 21.8 & 37.4 & 29.6 & 38.5 & 44.4 & 32.8 & 30.6 & 37.9 & 27.3 \\
\hline Moderada & 9.8 & 18.2 & 31.5 & 22.0 & 19.4 & 19.7 & 18.4 & 20.7 & 9.1 \\
\hline Severa & 0.8 & 10.1 & 7.4 & 8.8 & 5.6 & 11.5 & 0.0 & 3.4 & 9.1 \\
\hline Muy severa & 0.0 & 1.0 & 0.0 & 0.0 & 5.6 & 1.6 & 4.1 & 0.0 & 0.0 \\
\hline \multicolumn{10}{|l|}{ Puntaje de impactos $n=133$} \\
\hline Rango: mín.-máx. & $1-33$ & $1-9$ & $1-6$ & $1-6$ & $1-9$ & $1-9$ & $1-9$ & $1-6$ & $1-6$ \\
\hline Media (d.e.) & $7.95(7.20)$ & $2.41(1.66)$ & $2.52(1.50)$ & $2.35(1.45)$ & $2.64(2.00)$ & $2.49(1.79)$ & $2.00(1.65)$ & $2.07(1.22)$ & $1.91(1.47)$ \\
\hline
\end{tabular}

Tabla 2. Frecuencia y media de condiciones orales percibidas como causa de impactos bucales según sexo y nivel educativo en escolares de Piura, $(n=150)$.

\begin{tabular}{|c|c|c|c|c|c|c|c|c|}
\hline \multirow{3}{*}{$\begin{array}{l}\text { Condiciones bucales percibidas como causa de } \\
\text { impactos }\end{array}$} & \multirow{2}{*}{\multicolumn{2}{|c|}{ Frecuencia }} & \multicolumn{3}{|c|}{ Sexo } & \multicolumn{3}{|c|}{ Nivel Educativo } \\
\hline & & & \multirow{2}{*}{$\begin{array}{c}\begin{array}{c}\text { Varones } \\
(\mathbf{n}=\mathbf{8 9})\end{array} \\
\%\end{array}$} & \multicolumn{2}{|l|}{$\begin{array}{c}\text { Mujeres } \\
(n=61)\end{array}$} & \multirow{2}{*}{$\begin{array}{c}\begin{array}{c}\text { Primaria } \\
(\mathbf{n}=79)\end{array} \\
\%\end{array}$} & \multicolumn{2}{|l|}{$\begin{array}{l}\text { Secundaria } \\
\quad(n=71)\end{array}$} \\
\hline & $\mathrm{n}$ & $\%$ & & $\%$ & $\mathrm{p}^{*}$ & & $\%$ & $\mathrm{p} *$ \\
\hline Dolor de muela & 87 & 58.0 & 56.2 & 60.7 & 0.580 & 68.4 & 46.5 & 0.043 \\
\hline Diente sensible & 49 & 32.7 & 28.1 & 39.3 & 0.040 & 17.7 & 49.3 & 0.042 \\
\hline Diente cariado & 16 & 10.7 & 14.6 & 4.9 & 0.060 & 10.1 & 11.3 & 0.938 \\
\hline Caida de un diente de leche & 3 & 2.0 & 1.1 & 3.3 & 0.740 & 2.5 & 1.4 & 0.950 \\
\hline Fractura de diente permanente & 2 & 1.3 & 2.2 & 0.0 & n.a. & 2.5 & 0.0 & n.a. \\
\hline Forma o tamano de los dientes & 1 & 0.7 & 0.0 & 1.6 & n.a. & 1.3 & 0.0 & n.a. \\
\hline Posición de los dientes & 11 & 7.3 & 7.9 & 6.6 & 0.990 & 6.3 & 8.5 & 0.089 \\
\hline Sangrado de encías & 2 & 1.3 & 2.2 & 0.0 & n.a. & 2.5 & 0.0 & n.a. \\
\hline Otras nueve condiciones bucales + & 0 & 0.0 & 0.0 & 0.0 & n.a. & 0.0 & 0.0 & n.a. \\
\hline Todas las condiciones bucales & & & 59.33 & 40.67 & & 52.67 & 47.33 & \\
\hline $\begin{array}{l}\text { Media de condiciones percibidas como causa de } \\
\text { impacto }\end{array}$ & \multicolumn{2}{|c|}{$1.14(0.66)$} & $1.12(0.70)$ & $1.16(0.58)$ & $0.643 * *$ & $1.11(0.58)$ & $1.17(0.66)$ & $0.701 * *$ \\
\hline \multicolumn{9}{|l|}{ Media (d.e.) } \\
\hline $\begin{array}{l}\text { + Dientes separados, color de dientes, inflan } \\
\text { erupción de diente permanente, pérdida de d } \\
\text { n.a. No aplicable } \\
\text { d.e. Desviación Estándar } \\
\text { * Prueba Chi-Cuadrado } \\
\text { ** Prueba U de Mann-Whitney }\end{array}$ & $\mathrm{e} p$ & encías & & & & hal alie & ormaciór & boca, \\
\hline
\end{tabular}


Tabla 3. Extensión del impacto de las condiciones bucales sobre los desempeños diarios en escolares de 11 y 12 años de edad, de los distritos de Canchaque y San Miguel de El Faique según sexo y nivel educativo, Piura.

\begin{tabular}{|c|c|c|c|c|c|}
\hline & \multirow{2}{*}{$\mathbf{n}$} & \multirow{2}{*}{ Media (d.e.) } & \multicolumn{2}{|c|}{ Rango } & \multirow[b]{2}{*}{$\mathbf{p}$} \\
\hline & & & Mínimo & Máximo & \\
\hline \multicolumn{6}{|l|}{ Sexo } \\
\hline Mujeres & 61 & $3.07(2.11)$ & 0 & 8 & \multirow{2}{*}{0.698} \\
\hline Varones & 89 & $2.98(2.28)$ & 0 & 8 & \\
\hline \multicolumn{6}{|l|}{ Nivel educativo } \\
\hline Primaria & 79 & $2.94(2.25)$ & 0 & 8 & \multirow{2}{*}{0.500} \\
\hline Secundaria & 71 & $3.10(2.17)$ & 0 & 8 & \\
\hline Total & 150 & $3.01(2.20)$ & 0 & 8 & \\
\hline
\end{tabular}

d.e.= Desviacion Estándar

Prueba U de Mann-Whitney

Tabla 4. Frecuencia de desempeños diarios con impacto de condiciones bucales en escolares de 11 y 12 años, distritos de Canchaque y San Miguel de El Faique, según sexo y nivel educativo, Piura.

\begin{tabular}{|c|c|c|c|c|c|c|c|c|c|c|c|c|}
\hline \multirow{2}{*}{$\begin{array}{l}\text { Desempeños diarios con } \\
\text { impacto }\end{array}$} & \multicolumn{2}{|c|}{$(n=150)$} & \multicolumn{2}{|c|}{$\begin{array}{l}\text { Mujeres } \\
(n=61)\end{array}$} & \multicolumn{2}{|c|}{$\begin{array}{l}\text { Varones } \\
(\mathrm{n}=\mathbf{8 9})\end{array}$} & \multirow[t]{2}{*}{ p } & \multicolumn{2}{|c|}{$\begin{array}{c}\text { Primaria } \\
(n=79)\end{array}$} & \multicolumn{2}{|c|}{$\begin{array}{l}\text { Secundaria } \\
\qquad(\mathrm{n}=71)\end{array}$} & \multirow[t]{2}{*}{$\mathbf{p}$} \\
\hline & $\mathbf{n}$ & $\%$ & $\mathbf{n}$ & $\%$ & $\mathbf{n}$ & $\%$ & & $\mathbf{n}$ & $\%$ & n & $\%$ & \\
\hline Comer & 94 & 62.7 & 36 & 59.0 & 58 & 65.2 & 0.440 & 46 & 58.2 & 48 & 67.6 & 0.240 \\
\hline Hablar & 58 & 38.7 & 27 & 44.3 & 31 & 34.8 & 0.240 & 26 & 32.9 & 32 & 45.1 & 0.130 \\
\hline Limpiarse la boca & 90 & 60.0 & 33 & 54.1 & 57 & 64.0 & 0.220 & 45 & 57.0 & 45 & 63.4 & 0.420 \\
\hline Dormir & 37 & 24.7 & 16 & 26.2 & 21 & 23.6 & 0.710 & 20 & 25.3 & 17 & 23.9 & 0.840 \\
\hline Emocion & 61 & 40.7 & 26 & 42.6 & 35 & 39.3 & 0.690 & 34 & 43.0 & 27 & 38.0 & 0.530 \\
\hline Sonreir & 49 & 32.7 & 21 & 34.4 & 28 & 31.5 & 0.700 & 25 & 31.6 & 24 & 33.8 & 0.780 \\
\hline Estudiar & 30 & 20.0 & 12 & 19.7 & 18 & 20.2 & 0.930 & 14 & 17.7 & 16 & 22.5 & 0.460 \\
\hline Socializar & 33 & 22.0 & 16 & 26.2 & 17 & 19.1 & 0.300 & 22 & 27.8 & 11 & 15.5 & 0.070 \\
\hline Total & 133 & 88.7 & 57 & 93.4 & 76 & 85.4 & 0.130 & 72 & 91.1 & 61 & 85.9 & 0.310 \\
\hline
\end{tabular}

Prueba Chi cuadrado

Tabla 5. Severidad del impacto de las condiciones bucales sobre los desempeños en escolares de 11 y 12 años de edad, distritos de Canchaque y San Miguel de El Faique (Piura), según sexo y nivel educativo, (n=150).

\begin{tabular}{|c|c|c|c|c|c|c|c|c|c|c|c|c|c|}
\hline \multirow{2}{*}{$\begin{array}{l}\text { Desempeños } \\
\text { diarios con } \\
\text { impacto }\end{array}$} & \multirow[t]{2}{*}{$\begin{array}{c}\text { Media } \\
\text { (d.e.) }\end{array}$} & \multicolumn{2}{|c|}{ Rango } & \multicolumn{2}{|c|}{$\begin{array}{c}\text { Mujeres } \\
(n=61)\end{array}$} & \multicolumn{2}{|c|}{$\begin{array}{l}\text { Varones } \\
(n=89)\end{array}$} & \multirow[t]{2}{*}{$\mathbf{p}$} & \multicolumn{2}{|c|}{$\begin{array}{c}\text { Primaria } \\
(n=79)\end{array}$} & \multicolumn{2}{|c|}{$\begin{array}{l}\text { Secundaria } \\
\qquad(n=71)\end{array}$} & \multirow[t]{2}{*}{$\mathbf{p}$} \\
\hline & & & & Media & D.E. & Media & D.E. & & Media & D.E. & Media & D.E & \\
\hline Comer & $1.07(0.96)$ & 0 & 3 & 1.03 & 0.95 & 1.09 & 0.97 & 0.708 & 0.96 & 0.93 & 1.18 & 0.99 & 0.163 \\
\hline Hablar & $0.53(0.79)$ & 0 & 3 & 0.51 & 0.74 & 0.54 & 0.83 & 0.821 & 0.46 & 0.80 & 0.61 & 0.78 & 0.248 \\
\hline Limpiarse la boca & $0.84(0.84)$ & 0 & 3 & 0.70 & 0.76 & 0.93 & 0.89 & 0.102 & 0.80 & 0.87 & 0.89 & 0.82 & 0.517 \\
\hline Dormir & $0.37(0.77)$ & 0 & 3 & 0.34 & 0.70 & 0.39 & 0.81 & 0.696 & 0.42 & 0.83 & 0.32 & 0.69 & 0.427 \\
\hline Emocion & $0.56(0.80)$ & 0 & 3 & 0.59 & 0.80 & 0.54 & 0.80 & 0.708 & 0.56 & 0.75 & 0.56 & 0.86 & 1.000 \\
\hline Sonreir & $0.39(0.64)$ & 0 & 3 & 0.41 & 0.67 & 0.38 & 0.63 & 0.780 & 0.41 & 0.71 & 0.38 & 0.57 & 0.777 \\
\hline Estudiar & $0.25(0.56)$ & 0 & 2 & 0.25 & 0.54 & 0.26 & 0.58 & 0.914 & 0.25 & 0.59 & 0.25 & 0.53 & 1.000 \\
\hline Socializar & $0.30(0.65)$ & 0 & 3 & 0.36 & 0.71 & 0.26 & 0.61 & 0.358 & 0.35 & 0.66 & 0.24 & 0.64 & 0.303 \\
\hline Total & $0.54(0.49)$ & 0 & 3 & 0.52 & 0.47 & 0.55 & 0.50 & 0.861 & 0.52 & 0.51 & 0.55 & 0.46 & 0.439 \\
\hline
\end{tabular}




\section{DISCUSIÓN}

El estudio se realizó en un ámbito rural del norte del Perú, en Piura. No existen estudios previos sobre el impacto de las condiciones bucales en la CVRSB de escolares en ámbitos rurales del Perú. Se utilizó el Índice Child-OIDP en su versión validada en español para Perú, ya usada en poblaciones urbanas de Zapallal, Puente Piedra y Lima (11). La versión original del Índice Child-OIDP (8), está orientada a adolescentes con un promedio de 12 años de edad, etapa en que los niños ya tienen suficiente criterio para percibir incomodidades, manifestar sus percepciones y recordar eventos en el pasado $(2,8)$. Además, la OMS recomienda ésta edad como una de las edades importantes para el empleo de índices, comparación internacional y vigilancia epidemiológica en salud bucal (12).

En el presente estudio se halló una alta prevalencia del impacto de las condiciones bucales sobre la CVRSB de los escolares evaluados. El 88,7\% de ellos manifestó al menos un impacto en alguno de sus desempeños diarios en los tres meses previos al estudio. Esta prevalencia resulta semejante a la encontrada en otros estudios para adolescentes de 12 años de edad: en niños tailandeses (2), franceses (13), en dos estudios en niños brasileños $(14,15)$, en niños sudaneses (9), y en escolares peruanos de Zapallal, Lima (11). Estas altas prevalencias permitirían considerar que el impacto de las condiciones bucales en la CVRSB fue más frecuente en esta edad que en otros grupos etáreos, pues otros estudios con el OIDP en edades mayores, han mostrado prevalencias menores en ancianos de México (16), en adolescentes de 15 y 16 años de edad en Brasil (17), en adultos jóvenes en Tanzania (18), en adultos mayores en Tailandia (19) y en adultos mayores dentados y edéntulos en Grecia y Gran Bretaña (20).

La prevalencia de impactos hallada en escolares de ámbito rural en Piura fue mayor que la encontrada en el ámbito urbano de Zapallal, Lima (11); situación distinta a la encontrada en adultos, menor en la zona rural que en la zona urbana, en Granada, España (18).

Los escolares de Piura señalaron 8 de 17 Condiciones bucales, como causa de impacto en sus desempeños diarios. En orden de mayor a menor frecuencia, las condiciones fueron: dolor de muela, diente sensible, diente cariado, posición de los dientes, caída de un diente de leche, fractura de un diente permanente, sangrado de encías y forma o tamaño de los dientes. Las tres más frecuentes: dolor de muela, diente sensible y diente cariado, son síntomas frecuentes relacionados con caries dental avanzada, y corroborarían una alta prevalencia de impactos en relación con alta prevalencia de caries en el Perú: 86.64 de niños con caries a los 12 años y con un CPOD-12 de 3.67 (21). Otros estudios también han encontrado estas mismas condiciones como principales causas de impacto: jóvenes en Tanzania mencionaron caries dental (18), escolares en Brasil mencionaron diente sensible y dolor de muela (14), escolares de Sudan mencionaron dolor de muela como una causa frecuente (9) y escolares de Zapallal, Lima, Perú, refirieron dolor de muela como causa principal de los impactos (11). La forma o tamaño de los dientes, sangrado de encías y fractura de diente permanentes fueron los problemas bucales menos mencionados por los escolares de Piura como causa de impacto. No se hallaron diferencias significativas de las frecuencias de condiciones como causa de impacto en los desempeños entre los escolares según nivel educativo, posiblemente porque la diferencia entre los niveles educativos primaria-secundaria corresponde en este caso a solo un año. Constituyó un hallazgo interesante la diferencia significativa en la frecuencia de diente sensible como causa de impacto, mayor en mujeres que en varones, para lo cual no se tendría en este estudio una explicación pero que posteriores estudios podrían corroborar o descartar.

En los escolares de Piura, la prevalencia deimpactos más alta fue para el desempeño: comer $(62,7 \%)$, encontrándose concordancia con lo encontrado por Sánchez (11) en Zapallal - Lima, (48,0\%). En otros países se han encontrado prevalencias de impacto para comer tan bajas como 23,2\% en Gran Bretaña (22) y tan altas como $72,9 \%$ en Tailandia (2). En Piura, como en Zapallal (Lima) y en otros países, comer fue el desempeño con mayor frecuencia de impacto. Las condiciones bucales que impactan en la CVRSB varían de una región a otra, encontrándose algunos factores que podrían influir en ello, tales como la prevalencia de caries dental entre ellas, lo cual podría explicar tales diferencias. Las condiciones bucales de orden natural, como la caída de un diente de leche, son condiciones más homogéneas en cualquier población y su potencial como causa de impacto en la CVRSB parece ser menor. 
El promedio de severidad del impacto sobre todos los desempeños diarios, en los escolares de Piura, fue bajo $(0,54)$. Los desempeños: comer y limpiarse la boca tuvieron los promedios más altos (1,07 y 0,84 respectivamente), guardando coherencia con las condiciones bucales auto-percibidas más frecuentes: dolor de muela, diente sensible y diente cariado, como causa de impacto. La severidad de los impactos fue poca o muy poca en la mayoría de escolares, pero más de uno de cada diez escolares con impactos en los desempeños: comer, dormir y emoción manifestaron intensidades severa o muy severa en dichos desempeños y más de uno de cada 25 escolares con impactos en sonreír manifestó intensidad de impacto muy severa en dicho desempeño. Los escolares de Piura tuvieron mayor proporción de intensidades de impacto poco y muy poco en sus desempeños, y menor proporción de intensidades severa y muy severa, que los escolares de Tailandia (2) y de Zapallal, Piura (11).

La extensión del impacto tuvo un promedio de 3,01 desempeños impactados por escolar. Ningún escolar manifestó impacto sobre todos los desempeños. La mayoría de ellos informó impactos en uno a cuatro desempeños, siendo los más frecuentemente impactados: comer, limpiarse la boca y emoción. En Zapallal la extensión del impacto tuvo un promedio de 2,72 desempeños impactados (11).

El Índice Child-OIDP, que estima el impacto de las condiciones bucales en la CVRSB, tuvo un puntaje promedio global de 7,05 en los 150 escolares estudiados de Piura. Este promedio es cercano a lo hallado en escolares de algunas otras poblaciones: 7.7 en Lima (Zapallal) (11), 6.32 en Francia (13) y 7.1 en Brasil (15); aunque otros estudios hallaron promedios más altos: 8.85 en Tailandia (2) y 9.2 en Brasil (14). Las diferencias podrían corresponder a distintas características socio-geográficas.

Por desempeños, los mayores promedios del índice, fueron para: comer $(1,59)$, limpiarse los dientes $(1,43)$, emoción $(1,01)$ y hablar $(0,91)$; mientras que los menores promedios fueron para: estudiar y socializar.

Cuando se calculó el Índice global solo para los escolares con al menos un impacto (133 escolares) el promedio global aumentó a 7,95, y los promedios de todos los desempeños aumentaron, resultando los mayores promedios para: comer, hablar, dormir y socializar, duplicándose para: hablar y emoción; cuadruplicándose para dormir y socializar; y quintuplicándose para estudiar. Estos incrementos estarían indicando una configuración más ajustada del impacto en los desempeños en los 133 escolares con impactos.

Los hallazgos en el área rural en Piura, indican según sexo y según nivel educativo, que los escolares no tuvieron diferencias importantes en prevalencia de impactos y en severidad, intensidad y extensión de los impactos en los desempeños y en los puntajes del índice. Ello es semejante a los hallazgos en zona urbana de Zapallal, Lima (11). Aunque el Índice Child-OIDP de los escolares en Piura indica una calidad de vida con mayor impacto en los desempeños diarios de los escolares que en Zapallal, ello no revela diferencias importantes.

Una limitante del presente estudio es la muestra tomada por conveniencia, que por estar constituida sólo por los escolares que estuvieron presentes en las I.E. rurales durante el estudio, lo hace sólo válido para el grupo estudiado.

En conclusión, la prevalencia de escolares que señalaron algún impacto de las condiciones bucales en sus desempeños diarios fue alta $(88,7 \%)$. La severidad del impacto de las condiciones bucales sobre los desempeños fue leve. La intensidad de los impactos sobre los desempeños diarios fue de "muy poca intensidad" y "poca intensidad" para la mayoría de escolares. Los mayores promedios para el Índice Child-OIDP determinados en toda la muestra fueron para: comer y limpiarse la boca. El promedio más alto del Índice Child-OIDP sólo en escolares con algún impacto por desempeño, fue para: dormir. El estudio aporta información preliminar sobre calidad de vida relacionada a la salud bucal en escolares de 12 años de edad en ámbito rural del Perú, realidad sobre la cual no se ha contado con estudios previos.

\section{Correspondencia}

César E. del Castillo-López.

Departamento Académico de Odontología Social.

Facultad de Estomatología Roberto Beltrán, Universidad Peruana Cayetano Heredia.

Av. Honorio Delgado No 430, San Martín de Porres. Lima, Perú.

Correo electrónico: cesar.del.castillo.1@upch.pe 


\section{REFERENCIAS BIBLIOGRÁFICAS}

1. Sheiham A, Steele JG, Marcenes W, Tsakos G, Finch S, Walls AWG: Prevalence of impact of dental and oral disorders and their effects on eating among older people; a national survey in Great Britain. Community Dent Oral Epidemiol. 2001; 29:195-203.

2. Gherunpong S, Tsakos G, Sheiham A: The prevalence and severity of oral impacts on daily performances in Thai primary school children. Health Qual Life Outcomes. 2004: 2: 57.

3. Guyatt GH, Feeny DH, Patrick DL. Measuring HealthRelated Quality of Life. Ann Intern Med.1993; 118(8):622-9.

4. Schwartzmann L. Calidad de vida relacionada con la salud:Aspectos conceptuales. Cienc. enferm. [revista en laInternet]. 2003 Dic [citado 2014 Nov 13]; 9(2):09-21. Disponible en: http://www.scielo.cl/scielo.php?script= sci_arttext\&pid=S0717-95532003000200002\&lng=es. http://dx.doi.org/10.4067/S0717-95532003000200002.

5. Allen PF. Assessment of oral health related quality of life. Health Qual Life Outcomes. [Internet]. 2003 [citado 2014 Nov 13]; 1:40. Disponible en: http:// www.hqlo.com/content/1/1/40.

6. Adulyanon S, Sheiham A. Chapter 14. Oral impacts on daily performances. En: Slade GD, Editor. Measuring oral health and quality of life. Chapel Hill, NC: University of North Carolina; Dental Ecology 1997. p. 151-60.

7. Locker D. Measuring oral health: a conceptual framework. Community Dent Health. 1988; 5(1):3-18.

8. Gherunpong S, Tsakos G, Sheiham A. Developing and evaluating an oral health-related quality of life index for children; the CHILD-OIDP. Community Dent Health. 2004; 21(2):161-9.

9. Nurelhuda NM, Ahmed MF, Trovik TA, Astrom AN. Evaluation of oral health-related a quality of life among Sudanese schoolchildren using Child-OIDP inventory. Health Qual Life Outcomes. [Internet]. 2010 [citado 2014 Nov 13]; 2010,8:152. Disponible en: http://www.hqlo.com/content/8/1/152. Bernabé E, Sheiham A, Tsakos G. A comprehensive evaluation of the validity of Child-OIDP: further evidence from Peru. Community Dent Oral Epidemiol 2008; 36(4):317-25.

10. Sánchez P. Impacto de las condiciones orales sobre la calidad de vida en escolares de 11 y 12 años de edad de Zapallal-Puente Piedra. Lima. [Tesis de Maestría]. Lima: Facultad de Estomatología Perú. Universidad Peruana Cayetano Heredia; 2007.

11. WHO. Oral Health Surveys. Basic Methods. $4^{\text {th }}$ Ed. 1997.
12. Tubert-Jeannin S, Pegon-Machat E, Gremeau-Richard C, Lecuyer M-M, Tsakos G. Validation of a French version of the Child-OIDP index. Eur J Oral Sc 2005; 113(5): 355-62.

13.Castro R-AL, Cortes M-IS, Leao A-T, et.al. Child-OIDP index in Brazil: Cross-cultural adaptation and validation. Health Qual Life Outcomes. [Internet]. 2008 [citado 2014 Nov 13]; 2008. 6:68. 7. Disponible en http://www.hqlo.com/content/6/1/68.

14. Castro Rde A, Portela MC, Leão AT, de Vasconcelos MT. Oral health-related quality of life of 11- and 12-year-old public school children in Rio de Janeiro. Community Dent Oral Epidemiol. 2011; 39(4):336-44.

15. Sánchez-García S, Juárez-Cedillo T, Reyes-Morales H, Fuente-Hernández J, Solórzano-Santos F, GarcíaPeña C. Estado de la dentición y sus efectos en la capacidad de los ancianos para desempeñar sus actividades habituales. Salud Pública Mex. 2007; 49:173-81.

16. de Oliveira CM, Sheiham A. The relationship between normative orthodontic treatment need and oral healthrelated quality of life. Community Dent Oral Epidemiol. 2003; 31: 426-36.

17. Masalu JR, Astrom AN. Applicability of an abbreviated version of the oral impacts on daily performances (OIDP) scale for use among Tanzanian students. Community Dent Oral Epidemiol. 2003; 31:7-14.

18. Srisilapanan P, Sheiham A. The prevalence of dental impacts on daily performances in older people in Northen Thailand. Gerodontology. 2001; 18(2): 102-8.

19. Tsakos G, Marcenes W, Sheiham A. Cross-cultural differences in oral impacts on daily performance between Greek and British older adults. Community Dent Health. 2001; 18(4): 209-13.

20. Perú/Ministerio de Salud. Prevalencia nacional de caries dental, fluorosis del esmalte y urgencia de tratamiento en escolares de 6 a 8, 10, 12 y 15 años, Perú, 20012002. Lima: Oficina General de Epidemiología; 2005. Serie Informes Técnicos de Investigación Epidemiológica: $\mathrm{N}^{\mathrm{o}} 05 / 050$.

21. Yusuf H, Gherunpong S, Sheiham A, Tsakos G. Validation of an English version of the Child-OIDP Index, an oral health-related quality of life measure for children. Health Qual Life Outcomes. [Internet]. 2006 [citado 2014 Nov 13]; 2006,4-38. Disponible en: http://www.hqlo.com/content/4/1/38.

Recibido: 04/01/2014

Aceptado: 10/03/2014 